\title{
Palinopsia: a Review of the Literature
}

\author{
SUSAN J. STAGNO ${ }^{1}$ and TODD J. GATES ${ }^{2}$ \\ 'Departments of Psychiatry and Neurology, Cleveland Clinic Foundation, Cleveland, Ohio 44195, USA \\ ${ }^{2}$ Cleveland, Ohio
}

\begin{abstract}
The literature on palinopsia (visual perseveration) is reviewed, utilizing case reports of 46 patients who demonstrated this symptom. The most common etiologies for this symptom are space-occupying lesions, cerebral infarct, and seizure activity. The vast majority are due to central nervous system pathology occurring in the posterior (occipital or parieto-occipital) region, often in the right hemisphere. Proposed mechanisms for palinopsia are also discussed.
\end{abstract}

\section{Introduction}

Palinopsia derives from the Greek; palin meaning again and -opsis meaning vision. It is defined by Bender et al. (1968) as "the persistence or recurrence of visual images after the exciting stimulus object has been removed."

Palinopsia is an uncommon neuro-ophthalmic symptom. Despite its rarity, it has been described in a number of conditions including space occupying brain lesions (Ardila et al., 1987; Bender, 1968; Critchley, 1951; Jacobs, 1980; Lazaro, 1983; Patterson et al., 1985), cerebral infarctions (Ardila et al., 1987; Cleland et al., 1981; Gates et al., 1988; Kinsbourne and Warrington, 1963; Meadows and Munro, 1977; Michel and Troost, 1980), migraine cephalgia (Klee and Willanger, 1966) and ictal phenomena (Bender, 1968; Robinson and Watt, 1947; Swash, 1979). It has also been a symptom ascribed to drug intoxication, such as with mescaline (Critchley, 1951), and has been seen in metabolic disturbances such as hyperglycemia (Johnson and Loge, 1988). It is closely associated, and may occur with other neuro-ophthalmic symptoms including as cerebral polyopia (Bender, 1968; Kinsbourne and Warrington, 1963; Young et al., 1989), monocular diplopia (Ardila et al., 1987; Bender, 1968), metamorphopsia (Critchley, 1951; Young et al., 1989) and visual allesthesia (Ardila et al., 1987; Critchley, 1951; Jacobs, 1980). Because this symptom is rare and unusual, patients reporting it may be suspected of having a psychiatric illness such as hysteria or psychosis. Patients may also be hesitant to describe the symptom to the doctor for fear of being regarded as mentally ill.

This article will serve as a review of cases of palinopsia or visual perseveration reported in the English literature, a discussion of the mechanisms proposed to cause this symptom, and a caveat for clinicians to carefully evaluate patients with unusual visual symptoms or "visual hallucinations" to rule out organic causes. 


\section{Palinopsia: Description of the Symptom}

The term palinopsia actually has been used to describe a nonhomogenous group of visual responses. For example, the patient may see an item, such as a spoon, and upon shifting his visual focus, have the spoon immediately appear in his visual field again. It most often appears exactly as it had been seen originally, or may appear distorted (for example, smaller, changed in colour, etc.). Alternatively, the palinopic image may be delayed in its reappearance by minutes, hours, or sometimes days. Another form of palinopsia which has been described is "spreading" of a pattern or image. As an example, a person may look at a patterned wallpaper and see the pattern extending onto adjacent areas where the wallpaper does not exist. Another interesting form of palinopsia is the experience of superimposing a physical feature, such as a beard, onto the faces of everyone the patient sees. Palinopsia may also be experienced as a repetitive scene. For example, a patient may see an orderly bringing a tray of food to his bedside, and then re-experience the entire scene, including the movement, just as though it was happening again. One case report (Cleland et al., 1981) describes a patient who experienced accelerated movement of the perseverated image. Swash (1979) described two cases of visual perseveration occurring as a part of ictal activity in which the patients experienced attacks during which their vision became fixed on an object or image which would then be retained for several minutes. The false image or object would then gradually fade away, with the real external world becoming increasingly clear. He emphasized that this sort of phenomenon is different from palinopsia as described by Bender (1968), in that recurrence of a visual image represents inappropriate recall of an image from visual memory stores, whereas persistence of the image is due to failure of extinction of a visual image from consciousness.

This differs from Critchley's (1951) original definition of types of visual perseveration. Critchley felt that at least two terms were required to adequately describe these phenomena: visual perseveration in time, which he termed "paliopsia" "whereby the patient continues to see the object after it has been removed"; as opposed to visual perseveration in space, which he called "illusory visual spread" "when the patient continues to see the object in an interrupted--rather than continuous-fashion." He suggested alternative names for this might be visual extension, visual reduplication, or visual repetition. He regarded this type of visual perseveration as, at times, taking the form of metamorphopsia whereby objects appear elongated in one dimension only. His clinical example of the phenomenon of visual illusory spread was that of a woman who, when looking at a person with patterned clothing such as stripes or checks, would see the pattern extending over the person's face. Another example from the same patient was that she saw the iron railings enclosing the hospital extend across the road, and thought the taxi was charging through this barrier.

For the remainder of this article, the terms palinopsia and visual perseveration will be used interchangeably, unless otherwise specified.

The time interval between seeing an object or a scene and experiencing a visual recurrence is a controversial issue in terms of classifying the event as 
palinopsia. For example, Bender (1968) describes the case of a woman who clearly experienced classical symptoms of palinopsia but who also vividly saw a painting of her sister which had once hung in her living room but had been removed 10 years previously. Bender states, "The image of the painting of her sister seen 10 years previously might have been an hallucination rather than the remote possibility of a non-continuous palinopsia." Robinson and Watt (1947) described two patients wounded in military combat and who experience "hallucinations of remembered scenes" as an aura of their seizures that were caused by penetrating head injuries. These scenes involved experiences that had happened to the patients in the relatively distant past (weeks to months) and tended to relate to their experience of the injury (e.g. in the first case, the patient experienced the scene of the stretcher bearers coming to get him at the time of the injury, as well as other scenes; the second patient saw the events that occurred at the time of his injury on board ship). Of note is the fact that this paper was written in 1947, before Critchley (1951) had coined the term "paliopsia."

\section{Clinical Features of the Cases Reviewed}

Forty-six cases of palinopic experiences were reviewed (Ardila et al., 1987; Bender et al., 1968; Cleland et al., 1981; Critchley, 1951; Cummings et al., 1982; Gates et al., 1988; Jacobs, 1980; Jacome, 1985; Johnson and Loge, 1988; Joseph, 1986; Kinsbourne and Warrington, 1963; Klee and Willanger, 1966; Lazaro, 1983; Meadows and Munro, 1977; Michel and Troost, 1980; Patterson et al., 1985; Robinson and Watt, 1947; Swash, 1979; Young et al., 1989). Not all authors necessarily regarded their patients with visual perseveration as having palinopsia (Swash, 1979), as noted above but are included here for completeness.

Palinopsia is classically associated with a lesion occurring in the right posterior (occipital or parieto-occipital) hemisphere. Seventeen of the 46 cases reviewed were definite right hemisphere lesions, and five additional cases were most probably due to right hemisphere pathology. In 12 cases, the lesion was in the left posterior hemisphere, and one additional case most probably left. Four cases showed bilateral pathology, but in two of these cases, the lesions on the right side were most likely to be causing the visual symptoms described. In three cases, the laterality was unknown and in one case the patient was blind in the left eye. In some cases, such as migraine, laterality is unable to be ascertained.

In the vast majority of cases, the palinopic images occurred in a field of vision that was defective in some way: in ten patients a left homonymous hemianopia was found; in two, a left homonymous hemiachromatopsia; ten additional patients demonstrated left visual field cuts of some type (most typically, a quadrantanopia). Eight patients had right visual field disturbances. In only one patient (Jacome, 1985) was a bitemporal field cut noted, and this was an intermittent finding occurring on visual fixation in a patient with multiple sclerosis. Again, one patient was blind in the left eye, and another had impaired vision in the left eye. In six cases it is unknown 
whether visual field disturbances occurred; three of these were in patients with migraine headaches.

With respect to etiology, 16 cases were due to space occupying lesions: 11 were due to central nervous system (CNS) neoplasms (seven in the right hemisphere, four in the left); one due to abscess (right hemisphere); one to neurocysticercus with bilateral CNS lesions, but the area responsible for the palinopsia was more likely on the left; one due to an arterio-venous malformation on the right (with an epileptic focus seen on EEG); one due to bone chips (left hemisphere) and one to shell fragments (right hemisphere). In some patients with space occupying lesions, the palinopsia seemed to be seen in association with a possible seizure phenomenon due to irritation from the lesion, whereas in others it occurred in the setting of acute neurologic symptoms from the etiological lesion and were more likely due to disordered temporal synthesis of visual information (Michel and Troost, 1980). Ten cases were due to infarcts - six on the right, four on left. Six described visual perseveration or palinopic-like images occurring during seizure activityone with interictal epileptiform activity seen predominantly on the right, the five remaining on the left. Two patients developed their symptoms of palinopsia after having surgery in the right cerebral hemisphere. Another (Cummings, 1982) began experiencing palinopsia after needle biopsy of a mass in the right parieto-occipital area. One patient was blind in the left eye. Two unusual causes for the palinopsia were from bilateral optic neuritis in a patient with multiple sclerosis (Jacome, 1985), and another from contra coup damage from a head injury (Kinsbourne and Warrington, 1963). In two patients, no lesion could be identified, and in four cases the cause was either unknown or the description of the case was not complete enough to identify the etiology.

Palinopsia is a symptom of relatively short duration, measured in hours in a few cases, but more typically, several days, or a few weeks. However, in one case (Bender, 1968) the symptom occurred intermittently over 4 years, and toward the end of those 4 years, the symptom was nearly continuous. Another case in Bender's series lasted 2 years. The case described by Cummings (1982) occurred daily for 6 years. The patient with multiple sclerosis (Jacome, 1985) had symptoms of palinopsia more than 2 years.

Bender (1968) makes the point that palinopsia often occurred in conjunction with a clouded sensorium in the 12 patients he reported. Additional authors indicate that mental status may have been impaired while in other reports the patients had no impairment of their mental state. Bender (1968) also suggests that the patient's emotional experience was not one of concern or anxiety, whereas the patient reported by Gates et al. (1988) was quite anxious and agitated, lending to the supposition that the patient's symptoms were from a psychiatric cause.

\section{Proposed Mechanisms}

Why does this symptom occur commonly with right sided lesions? Just as other neurologic functions are served by a specific area or hemisphere within 
the brain, it has also been suggested that the right parietal cortex plays a specific role in the intellectual processing of visual data (Basso et al., 1973). Interestingly, many reports of formed hallucinations occurring secondary to CNS lesions are also frequently seen in patients with right hemisphere defects (Lance, 1976).

In his classic paper, Bender (1968) suggests four possible mechanisms to explain palinopsia. They include (1) visual after-sensations; (2) sensory seizures; (3) hallucinations; and (4) psychogenic elaborations or fantasies. We shall examine each of these possibilities in more detail.

The concept of visual after-sensations, or the idea that palinopsia is an exaggeration of a normal visual process is appealing. In fact, Blythe $e t$ al. (1986) presented a case of a young woman who began having multiple neurologic complaints during her two pregnancies, and after the birth of the second child, began having visual symptoms consisting of white spots "like fire flies" brought on by head movement or bright light; white objects appearing brighter than before; and prolonged after images when she looked at bright lights or after flash photography that "lasted for tens of minutes." They draw the analogy of this patient's case to the symptom of palinopsia. Because this patient had these visual symptoms for a prolonged period of time, the investigators were able to carry out numerous tests over time. Unlike most patients with palinopsia, this young woman did not have a visual field defect. Her electroencephalogram (EEG) showed a minor left temporal lobe abnormality consisting of theta activity with occasional sharp waves. A definitive diagnosis was never established, but the possibilities of multiple sclerosis or systemic lupus erythematosis with cerebral complications were entertained. She was treated with low dose carbamazepine (300 mg per day) with improvement but not resolution of her visual symptoms.

Bender (1968) discusses the similarities and differences between palinopsia and normal after-images in detail, and the reader is referred to this excellent article. In summary, he points out that after-images are most prominently experienced in situations where the object is very bright or intense (as when a flash bulb is ignited), and this is typically not the case with palinopsia. Further, after-images are regarded by most authors as being retinal in origin. The overwhelming majority of cases of palinopsia are the result of lesions in the posterior cerebral hemisphere. An additional clinical feature is that after-images typically appear as complementary colours when viewed against a light background, whereas palinopic images are usually original colours.

Sensory seizures have also been another proposed mechanism for the production of palinopsia. In addition to clinical case descriptions, which will be considered in more detail, cortical stimulation studies done in patients being evaluated for surgery of epilepsy, show that visual experiences similar to palinopsia may occur (Penfield, 1963). This lends support to the notion than an irritative epileptic focus may result in a "replay" of past visual experiences (Jacobs, 1980).

Whether or not palinopic images should be regarding as "hallucina- 
tions" depends on the definition you wish to use. Lessell (1990) has defined a visual hallucination as a symptom in which the patient claims to see something that is not evident to others in the same environment. Using this definition, palinopsia qualifies. Lessell (1990) further defines three types of visual hallucinations (1) migranous; (2) ictal (resulting from focal anomalous brain discharges); and (3) release hallucinations (consequent to reduction of the normal visual input to the brain). All three types have been described with palinopsia. Klee and Willanger (1966) describe palinopsia occurring in association with migraine. Several authors, including Bender (1968), Critchley (1951), Johnson and Loge (1988), Swash (1979), and Robinson and Watt (1947), report palinopsia occurring as an ictal event. Other cases, in which a neoplasm or other space occupying lesion was present, are described in which the palinopic events are ictal-like in nature (Meadows and Munro, 1977; Jacobs, 1980). Cummings et al. (1982) suggest that "palinopsia is a unique type of release hallucination in which the exciting stimulus occurred in the recent past." They conclude this based on several similarities between palinopsia and release hallucinations. These include: both types of patients have lesions that cause visual field defects and interrupt cortical visual processing; visual hallucinations of a non-ictal type may occur in patients with palinopsia; and there is a predominance of right posterior lesions in both types of patients. The patient reported by Cummings et al. (1982) had abnormal pattern-reversal visual evoked potentials (PVEP) both during episodes of palinopsia, which showed abnormal wave forms that varied considerably between averages, as well as abnormal PVEP between palinopic episodes. At these times, the P100 component showed abnormally prolonged latencies in both eyes. However, several EEGs were obtained while the palinopsia was occurring and the records showed no change from the resting pattern.

While the possible "psychogenic" cause of such a symptom must be considered in the differential diagnosis, this is a very unlikely cause of palinopsia. Only one paper describes palinopsia occurring in a patient with psychiatric illness (Joseph, 1986). This patient, however, displayed many neuropsychiatric symptoms, and suffered from Cotard's Syndrome (the delusion of being dead) as well as Capgras' Syndrome (the delusion of imposters or doubles) for which an organic basis has been suggested (Berson, 1983). This patient had no demonstrable CNS lesion on imaging studies but had neuropsychological testing revealing diffuse dysfunction, but with bifrontal and anterior right hemisphere predominance. The author suggests that "a unique neuroanatomical insult may explain the clinical presentation of the patient in this report." One other patient (Kinsbourne and Warrington, 1963) suffered from depression and had overdosed on barbiturates. During his psychiatric admission, however, he suffered a left occipital infarct causing his palinopsia.

Lastly, palinopsia has been likened to eidetic imagery; that is, the voluntary production of a vivid and detailed visual image from memory. Again, given that the reported cases all occurred in conjunction with CNS disease, and patients seemed not to be producing the symptoms by will, this 
also seems unlikely. Additional evidence in patients with migraine headaches is that these patients were often able to reproduce many of their visual symptoms, which normally occurred only in conjunction with the headache, with monocular fixation at a time separate from the headache. They were not able to reproduce the symptoms of palinopsia.

While all cases of palinopsia may not have the same underlying etiologic mechanism, the commonality in the occurrence of palinopsia in the majority of cases seems to be due to a cortical release phenomenon with interruption of the afferent pathways from the primary visual cortex to the visual association cortex. This "disconnection" or "deafferentation" may result in a loss of inhibitory neural influence and hyperactivity in the cortical association areas resulting in the prolongation or repetition of the visual image (Kinsbourne and Warrington, 1963; Lazaro, 1983; Patterson et al., 1985).

\section{Conclusion}

Palinopsia is an unusual visual symptom that has been reported in a variety of clinical settings in which the central nervous system is affected. Because of its typically transient and bizarre nature, it is likely to be under-reported. Patients may be reluctant to describe these strange symptoms and clinicians may not ask for detailed descriptions in patients complaining of "hallucinations" or other odd visual phenomena.

When palinopsia does occur, however, the reason for its occurrence is usually ominous and clearly is indicative of cerebral disease. These patients require careful and thorough evaluation, to rule out neoplasm or other space-occupying lesions, seizures which may be from multiple etiologies, and cerebral infarcts as the most likely causes.

\section{Acknowledgements}

The authors wish to acknowledge Paul A. Stagno, M.D., Ph.D. for his editorial comments and support; Harold H. Morris, III, M.D. for his helpful review; and Ms Marianne Newhouse for helping to make time available to work on this paper.

\section{References}

Ardila, A., Botero, M. and Gomez, J. (1987). Palinopsia and visual allesthesia. International Journal of Neuroscience, 32, 775-782.

Basso, A., DeRenzi, E., Fagliono, P., Scotti, G. and Spinnler, H. (1973). Neuropsychological evidence for the existence of cerebral areas critical to the performance of intelligence tasks. Brain, 96, 715-728.

Bender, M. B., Feldman, M. and Sobin, A. J. (1968). Palinopsia. Brain, 91, 321-338.

Berson, R. J. (1983). Capgras' Syndrome. American Fournal of Psychiatry, 140, 969-978.

Blythe, I. M., Bromley, J. M., Ruddock, K. H., Kennard, C. and Traub, M. (1986). A study of systematic visual perseveration involving central mechanisms. Brain, 109, 661-665. 
Cleland, P. G., Saunders, M. and Rossler, R. (1981). An unusual case of visual perseveration. Fournal of Neurology, Neurosurgery and Psychiatry, 44, 262-263.

Critchley, M. (1951). Types of visual perseveration: "Paliopsia" and "illusory visual spread". Brain, 74, 267-299.

Cummings, J. L., Syndulko, K., Goldberg, Z. and Treiman, D. M. (1982). Palinopsia reconsidered. Neurology (Ny), 32, 444-447.

Gates, T.J., Stagno, S.J. and Gulledge, A. D. (1988). Palinopsia posing as a psychotic depression. British Journal of Psychiatry, 153, 391-393.

Jacobs, L. (1980). Visual allesthesia. Neurology, 30, 1059-1063.

Jacome, D. E. (1985). Palinopsia and bitemporal visual extinction on fixation. Annals of Ophthalmology, 17, 251-252, and 257.

Johnson, S. F. and Loge, R. V. (1988). Palinopsia due to nonketotic hyperglycemia. Western Journal of Medicine, 148, 331-332.

Joseph, A. B. (1986). Cotard's syndrome in a patient with coexistent Capgras's syndrome, syndrome of subjective doubles, and palinopsia. Journal of Clinical Psychiatry, 47, 605-606.

Kinsbourne, M., and Warrington, E. K. (1963). A study of visual perseveration. Fournal of Neurology, Neurosurgery and Psychiatry, 26, 468-475.

Klee, A. and Willanger, R. (1966). Disturbances of visual perception in migraine: Review of the literature and a report of eight cases. Acta Neurologica Scandinavica, 42, 404-414.

Lance, J. W. (1976). Simple formed hallucinations confined to the area of a specific visual field defect. Brain, 99, 719-734.

Lazaro, R. P. (1983). Palinopsia: Rare but ominous symptom of cerebral dysfunction. Neurosurgery, 13, 310-313.

Lessell, S., Lessell, I. M. and Glaser, J. S. (1990). Topical diagnosis: Retrochiasmal visual pathways and higher cortical function. In "Neuroophthalmology" (Ed. J. S Glaser) 2nd edition. Lippincott, Philadelphia, pp. 213-238.

Meadows, J. C. and Munro, S. S. F. (1977). Palinopsia. Journal of Neurology, Neurosurgery and Psychiatry, 40, 5-8.

Michel, E. M. and Troost, B. T. (1980). Palinopsia: Cerebral localization with computed tomography. Neurology, 30, 887-889.

Patterson, M. C., Bunce, I. H. and Eadie, M.J. (1985). Cerebral abscess in leukaemia: An unusual presentation of a rare complication. Clinical and Experimental Neurology, 21, 257262.

Penfield, W. and Perot, P. (1963). The brain's record of auditory and visual experience. Brain, 86, 595-696.

Robinson, P. K. and Watt, A. C. (1947). Hallucinations of remembered scenes as an epileptic aura. Brain, 70, 440-448.

Swash, M. (1979). Visual perseveration in temporal lobe epilepsy. Journal of Neurology, Neurosurgery and Psychiatry, 42, 569-571.

Young, W. B., Heros, D. O., Ehrenberg, B. L. and Hedges, T. R. (1989). Metamorphosia and palinopsia: Association with periodic lateralized epileptiform discharges in a patient with malignant astrocytoma. Archives of Neurology, 46, 820-822. 


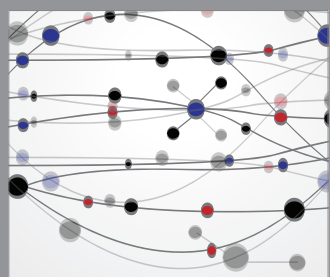

The Scientific World Journal
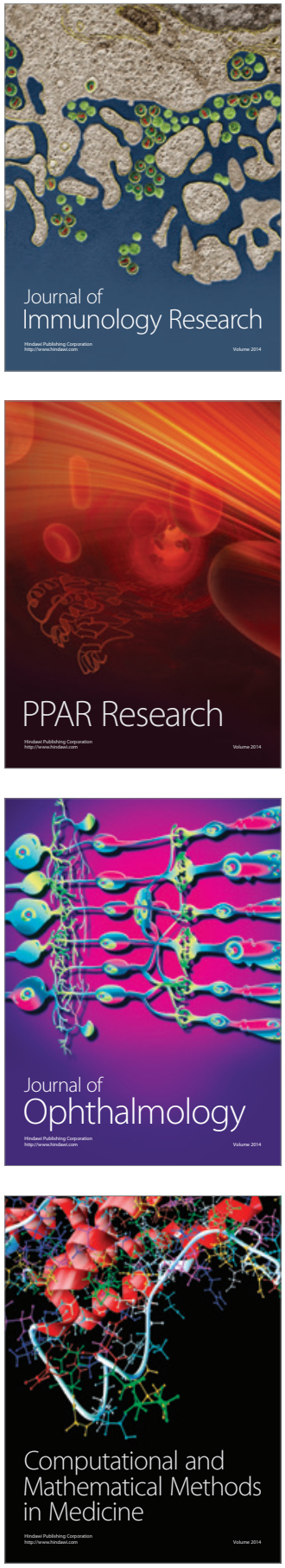

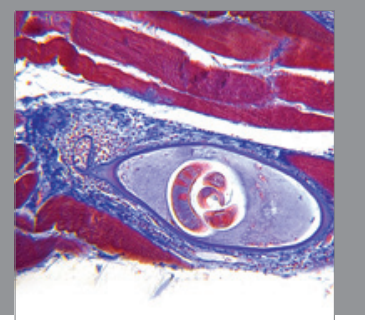

Gastroenterology

Research and Practice
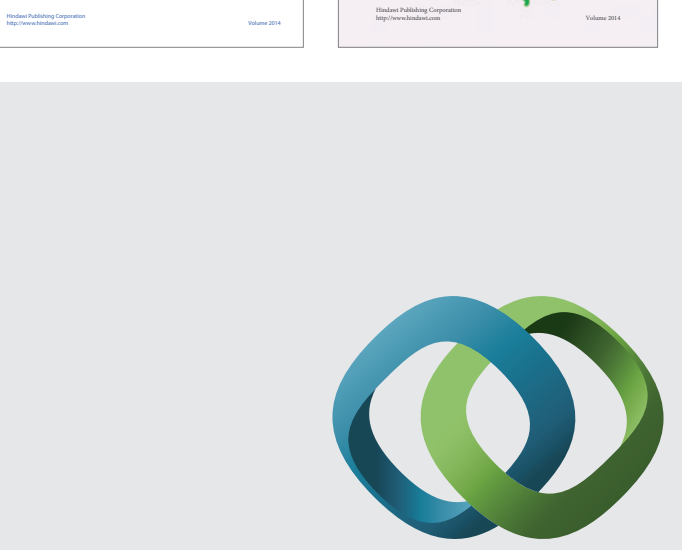

\section{Hindawi}

Submit your manuscripts at

http://www.hindawi.com
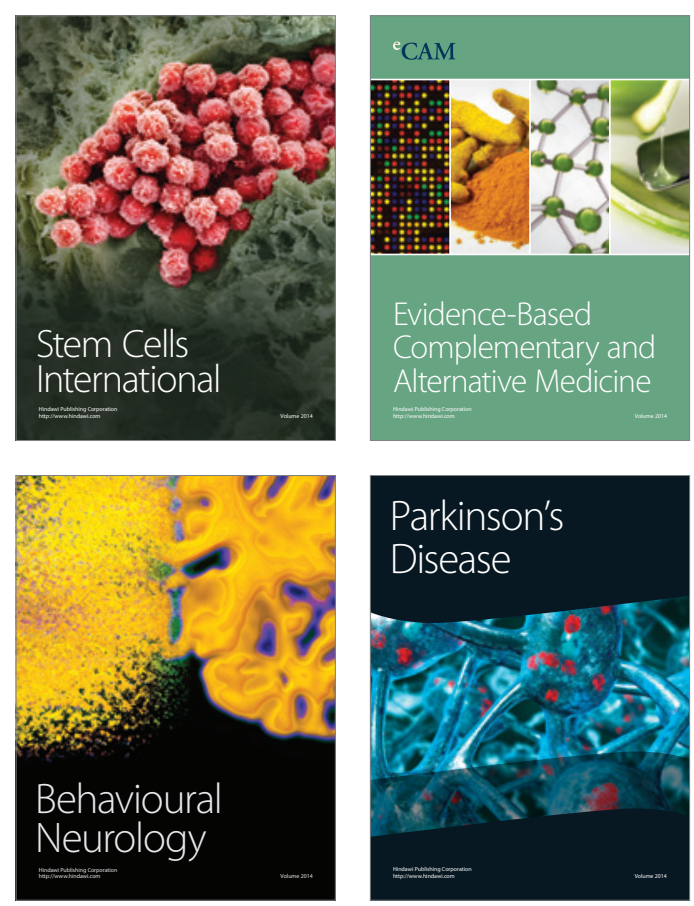

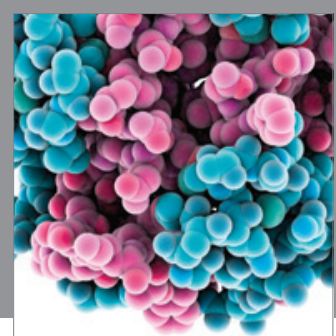

Journal of
Diabetes Research

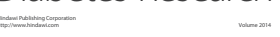

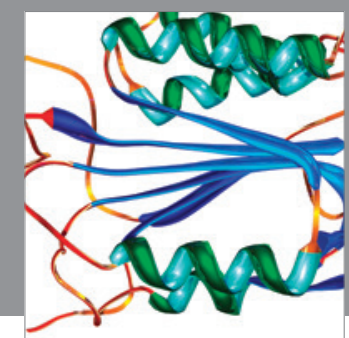

Disease Markers
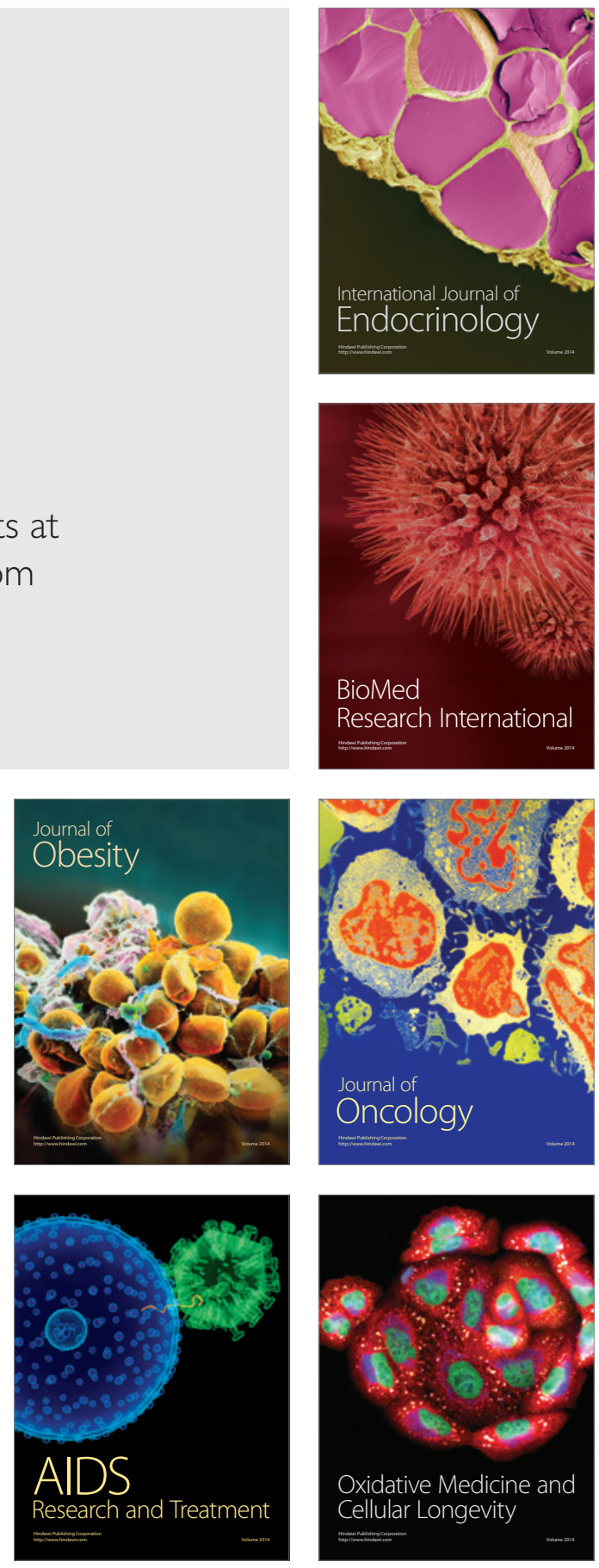